Annals of Pure and Applied Mathematics

Vol. 16, No. 1, 2018, 151-169

ISSN: 2279-087X (P), 2279-0888(online)

Published on 9 January 2018

Annals of

www.researchmathsci.org

DOI: http://dx.doi.org/10.22457/apam.v16n1a17

Pure and Applied

Mathematics

\title{
Effect of Hall Current in Oscillatory Flow of a Couple Stress Fluid in an Inclined Channel
}

\author{
Nirmala P. Ratchagar ${ }^{1}$, V. Balakrishnan ${ }^{2}$ and R. Vasanthakumari ${ }^{3}$ \\ ${ }^{1}$ Department of Mathematics, Annamalai University, Tamilnadu, India. \\ E-mail: nirmalapasala@yahoo.co.in \\ ${ }^{2}$ Department of Mathematics, Tagore Arts College, Puducherry, India. \\ E-mail: prasaanthbala@yahoo.com \\ ${ }^{3}$ Department of Mathematics, Kanchimamunivar Centre for Post Graduate Studies \\ Puducherry, India. \\ E-mail: vasunthara1@gmail.com
}

Received 22 December 2017; accepted 2 January 2018

Abstract. The effects of Hall current on oscillatory flow of a couple stress fluid in an inclined channel of blood has been considered. The closed form solutions for the velocity, temperature and concentration fields are obtained analytically and then evaluated numerically for different values of parameters using Mathematica, appearing in these equations. To have a better insight of the problem the variations of the physical quantities with flow parameters are shown graphically. By introducing a critical Magnetic field, the limit for Magnetic field with Hall current is also discussed.

Keywords: Hall current, oscillatory flow, couple stress fluid, MHD, inclined channel.

AMS Mathematics Subject Classification (2010): 76S05, 76W05

\section{Nomenclatures:}

$B_{o} \quad$ External magnetic field

$g \quad$ Gravitational acceleration

Gr Grashof number

Gc Modified Grashof number

$H \quad$ Hartmann number

K Permeability factor

$N \quad$ Thermal radiation parameter

$\mathrm{Nu} \quad$ Nusselt number

$p \quad$ Pressure

Pe Peclet number

$q \quad$ Radiative heat flux

Re Reynolds number

$k \quad$ Thermal conductivity

$t \quad$ Time

T, $C \quad$ Fuid temperature and

concentration

u Axial velocity

$(x, y) \quad$ Space coordinates

$\alpha \quad$ Angle of inclination

$C_{p} \quad$ Specific heat at a constant pressure

$\beta \quad$ Coefficient of volume expansion due to temperature

$\eta \quad$ Coefficient of couple stress

$\gamma \quad$ Couple stress parameter

$\mu \quad$ Dynamic viscosity

$v \quad$ Kinematic viscosity

$\sigma \quad$ Conductivity of the medium

$w \quad$ Angular frequency 
Nirmala P. Ratchagar, Balakrishnan and Vasanthakumari

$\begin{array}{ll}\rho & \text { Fluid density } \\ m & \text { Hall current parameter } \\ T_{0}, T_{\mathrm{w}} & \begin{array}{l}\text { Lower and upper wall } \\ \text { temperature }\end{array} \\ C_{0}, C_{\mathrm{w}} & \begin{array}{l}\text { Lower and upper wall } \\ \text { concentration }\end{array} \\ S c & \text { Schmidt number }\end{array}$

\section{Introduction}

Understanding the physics of oscillating (or transcient) flow of complex fluids in small channels is of fundamental interest for many biological and industrial processes, e.g. the quasi-periodic blood flow in the cardiovascular system can be described by the frequency components of the pressure and flow rate pulses. Many vascular diseases are associated with disturbances of the local flow conditions in the blood vessels. Various studies on oscillatory flow, experimental as well as theoretical, have been carried out by many researchers. A steady and transient solution describing the flow of a viscous fluid at small and large times by the Laplace transform method was presented by Erdogam [5]. Vajravelu and Rivera [24] obtained uniformly valid solutions for the hydromagnetic flow at both moving plate and an oscillating plate. Makinde and Mhone [9] investigated the combined effect of a transverse magnetic field and radiative heat transfer to unsteady flow of a conducting optically thin fluid through a channel filled with saturated porous medium and non-uniform walls temperature. Ali et al. [1] analysed hydromagnetic flow and heat transfer of a Jeffery fluid over an oscillating stretching surface and Nisat Nowroz Anika et.al [13] studied the Numerical treatment of MHD on unsteady Magnetohydrodynamics Fluid flow past an infinite rotating vertical porous plate with heat transfer considering the Hall current effect. Aamir et al. [2] studied a twodimensional oscillatory flow inside a rectangular channel for Jeffrey fluid with small suction and investigated the viscoelastic behavior of non-Newtonian fluids subject to time harmonic oscillations.

The study of couple stress fluid is very useful in understanding various physical problems because it possesses the mechanism to describe rheological complex fluids such as liquid crystals and human blood. By couple stress fluid, we mean a special case of non-Newtonian fluids. In further investigation many authors have assumed blood to be a suspension of spherical rigid particles (red cells), and this suspension of spherical rigid particles will give rise to couple stresses in a fluid.

The theory of couple stress was first developed by Stokes [22] which represents the simplest generalization of classical theory. He had proposed Linearized constitutive equations for force and couple stresses in fluids and solved a series of boundary-value problems to indicate the effects of couple stresses as well as for various material constants. A simple mathematical model depicting blood flow in the capillary is developed by Pal et al. [16], with an emphasis on the permeability property of the blood vessel, approximated as cylindrical tube with a permeable wall, based on Starling's hypothesis. Effect on the flow of blood by applying external magnetic field was studied by Eldabe et al. [6] by considering the flow is between two parallel fixed porous plates, which is the major consideration of this work. The couple stress fluid flow through porous medium has been studied by many researchers, e.g., Hiremath and Patil [7] 


\section{Effect of Hall Current in Oscillatory Flow of a Couple Stress Fluid in an Inclined Channel}

investigated the natural convection oscillatory flow of a couple stress fluid through porous medium. Ogulu [14] obtained the effects of radiative heat transfer and oscillatory temperature on couple stress fluid thermal convection in a porous medium. Sarojini et al. [18] investigated an MHD flow of a couple stress fluid through a porous medium in a parallel plate channel in the presence of the effect of inclined magnetic field. Md. Saidul Islam et.al [11] examined the steady two-dimensional MHD free convection and mass transfer flow past through an inclined plate with heat generation, and the MHD free convection fluid flow with the Soret effect on the combined heat and mass transfer in a rotating system was analysed by Rahman et al. [10]. On taking into account of the Hall current, an analysis of a generalized MHD Couette flow, is presented by Soundalgekar and Uplekar [21]. An analytical study on the oscillatory hydromagnetic flow of a viscous, incompressible, electrically-conducting, non-Newtonian fluid in an inclined, rotating channel with non-conducting walls, incorporating couple stress effects was presented by Sahin et al. [3]. Syamala et al. [23] discussed the steady hydro magnetic flow of a couple stress fluid in a parallel plate channel through a porous medium under the influence of a uniform inclined magnetic field inclined at an angle with the normal to the boundaries. Seth et al., [19] have investigated the effects of Hall current and rotation on unsteady MHD Couette flow of a viscous incompressible electrically conducting fluid in the presence of an inclined magnetic field and reported that the Hall current and rotation tend to accelerate fluid velocity in both the primary and secondary flow directions.

Magnetic field has retarding influence on the fluid velocity and the angle of inclination of magnetic field has accelerating influence on the fluid velocity. Under the influence of a uniform transverse magnetic field taking hall current into account the unsteady flow of an incompressible viscous fluid in a rotating parallel plate channel bounded on one side by a porous bed was presented by Veera Krishna and Jagdish Prakash [25] and the unsteady MHD free convection flow of an incompressible electrically conducting fluid by Veera Krishna and Swarnalathamma [26].

When an electrical current passes through a sample placed in a magnetic field, a potential proportional to the current and to the magnetic field is developed across the material in a direction perpendicular to both the current and to the magnetic field. This effect is known as the Hall effect, and is the basis of many practical applications and devices such as magnetic field measurements, and position and motion detectors. Effect of hall current on MHD flow fluid have been extensively studied by many authors. For e.g., such effect on nanofluid beyond boundary layer flow over rotating channel by Md. Abdel-Wahed and Md. Akl [12], the effects of Hall current, rotation and Soret number on an unsteady MHD natural convection flow with heat and mass transfer of a viscous, incompressible, electrically conducting fluid by Singh and Reena Pathak [20], the effect of mass transfer and Hall current on unsteady MHD flow of a viscoelastic fluid in a porous medium by Omokhuale and Onwuka [15], the effect of mass transfer and Hall current on unsteady MHD flow of a viscoelastic fluid in a porous medium by Khem Chand et al., [8], and the effects of Hall current, rotation and Soret number on an unsteady MHD natural convection flow with heat and mass transfer of a viscous, incompressible, electrically conducting fluid by Sarma and Pandit [17] are few among them. 
Nirmala P. Ratchagar, Balakrishnan and Vasanthakumari

In the present paper, the blood is represented by a couple stress fluid and we investigate the effect of hall current in oscillatory flow in an inclined channel. The closed form solutions for the velocity, temperature and concentration fields are obtained analytically and the software 'Mathematica' is used to obtain the numerical values for the different parameters appearing in the equations used in tis work. To have a better insight of the problem the variations of the physical quantities with flow parameters are plotted using Origin software. In the following sections, the problem formulated, solved, and the pertinent results are discussed.

\section{Mathematical formulation}

We consider the flow of an incompressible, viscous and electrically conducting couple stress fluid flowing through an inclined angle $\alpha$ under the influence of externally applied homogeneous magnetic field $B_{0}$ and radiative heat transfer as shown in the Figure 1. It is assumed that the fluid has small electrical conductivity and the electromagnetic force produced is very small. Assuming a Boussinesq incompressible couple stress fluid, the equations governing the motion for our model are as follows:

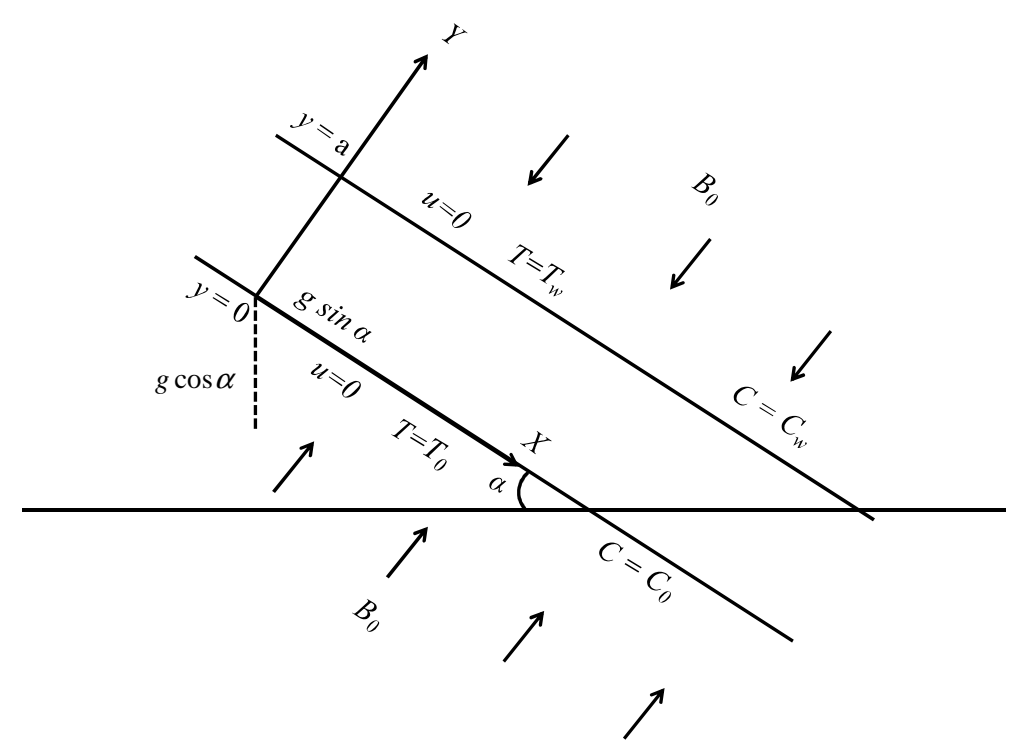

Figure 1: Geometry of the problem

The momentum, heat transfer and concentration equations are considered for our model in the form,

$$
\begin{aligned}
& \frac{\partial u}{\partial t}=\frac{-1}{\rho} \frac{\partial p}{\partial x}+v \frac{\partial^{2} u}{\partial y^{2}}+\eta \frac{\partial^{4} u}{\partial y^{4}}-\frac{v}{K} u-\frac{\sigma B_{0}^{2} u}{\rho\left(1+m^{2}\right)}+g \beta\left(T-T_{0}\right) \sin \alpha+g \beta_{c}\left(C-C_{0}\right) \sin \alpha \\
& 0=\frac{-1}{\rho} \frac{\partial p}{\partial y}
\end{aligned}
$$


Effect of Hall Current in Oscillatory Flow of a Couple Stress Fluid in an Inclined Channel

$\frac{\partial T}{\partial t}=\frac{k}{\rho c_{p}} \frac{\partial^{2} T}{\partial y^{2}}-\frac{1}{\rho c_{p}} \frac{\partial q}{\partial y}$
$\frac{\partial C}{\partial t}=D \frac{\partial^{2} C}{\partial y^{2}}-K_{c}^{\prime}\left(C-C_{0}\right)$

where $m=\frac{\sigma B_{0}}{e n}$ is the Hall parameter, $e$ is the electric charge, $\mathrm{n}$ is the number of density of electrons. The boundary conditions are,

$$
\begin{gathered}
u=0, T=T_{w}, C=C_{w}, \frac{\partial^{2} u}{\partial y^{2}}=0, \text { at } y=a \\
u=0, T=T_{0}, C=C_{0}, \frac{\partial^{2} u}{\partial y^{2}}=0, \text { at } y=0
\end{gathered}
$$

where, the meanings of all the symbols appearing in the equations are listed in the nomenclature. The fluid is observed to a optically thin with a relatively low density and radiative heat flux is given in Cogley et al. [4] by

$\frac{\partial q}{\partial y}=4 b^{2}\left(T_{0}-T\right)$

where, $b$ is the mean radiation absorption coefficient.

The following dimensionless variables and parameters are used:

$$
\begin{aligned}
& \bar{x}=\frac{x}{a}, \bar{y}=\frac{y}{a}, \bar{u}=\frac{u}{a}, \bar{p}=\frac{a p}{\rho v U}, \theta=\frac{T-T_{0}}{T_{w}-T_{0}}, \phi=\frac{C-C_{0}}{C_{w}-C_{0}}, \operatorname{Re}=\frac{U a}{v}, \\
& \bar{t}=\frac{t U}{a}, H^{2}=\frac{a^{2} \sigma B_{0}^{2}}{\rho v}, D a=\frac{K}{a^{2}}, S^{2}=\frac{1}{D a}, P e=\frac{U a \rho c_{p}}{k}, S_{c}=\frac{U a}{D}, \\
& N^{2}=\frac{4 b^{2} a^{2}}{k}, G r=\frac{a^{2} g \beta\left(T_{w}-T_{0}\right)}{v U}, G c=\frac{a^{2} g \beta_{c}\left(C_{w}-C_{0}\right)}{v U}, \gamma^{2}=\frac{\eta}{v a^{2}}, \\
& K_{c}=K_{c}^{\prime} \frac{a^{2}}{D}
\end{aligned}
$$

where $U$ is the flow mean velocity and $\mathrm{D}$ is the molecular diffusivity.

The dimensionless governing equations together with the appropriate boundary conditions ( neglecting the bars for clarity) can be written as

$$
\begin{aligned}
& \operatorname{Re} \frac{\partial u}{\partial t}=-\frac{\partial p}{\partial x}+\frac{\partial^{2} u}{\partial y^{2}}+\gamma^{2} \frac{\partial^{4} u}{\partial y^{4}}-\left(S^{2}+\frac{H^{2}}{1+m^{2}}\right) u+G r \sin \alpha \theta+G c \sin \alpha \phi \\
& 0=-\frac{\partial p}{\partial y} \\
& P e \frac{\partial \theta}{\partial t}=\frac{\partial^{2} \theta}{\partial y^{2}}+N^{2} \theta \\
& S c \frac{\partial \phi}{\partial t}=\frac{\partial^{2} \phi}{\partial y^{2}}-K c \phi
\end{aligned}
$$


Nirmala P. Ratchagar, Balakrishnan and Vasanthakumari

And the boundary conditions (5) and (6) becomes

$u=0, \theta=1, \phi=1, \frac{\partial^{2} u}{\partial y^{2}}=0$, at $y=1$

$u=0, \theta=0, \phi=0, \frac{\partial^{2} u}{\partial y^{2}}=0$ at $y=0$

In order to solve equations (9)-(12) for purely oscillatory flow, let,

$-\frac{\partial p}{\partial x}=B e^{i w t}, u(y, t)=u_{f}(y) e^{i w t}, \theta(y, t)=\theta_{f}(y) e^{i w t}, \phi(y, t)=\phi_{f}(y) e^{i w t}$

where $B$ is a constant and $w$ is the frequency of the oscillation. Substituting the above equations (15) into equations (9)-(12), we obtain:

$\gamma^{2} \frac{\partial^{4} u_{f}}{\partial y^{4}}+\frac{\partial^{2} u_{f}}{\partial y^{2}}-Z_{1} u_{f}=B-G r \sin \alpha \theta_{f}-G c \sin \alpha \phi_{f}$

$\frac{\partial^{2} \theta_{f}}{\partial y^{2}}+m_{1}^{2} \theta_{f}=0$

$\frac{\partial^{2} \phi_{f}}{\partial y^{2}}-m_{2}^{2} \phi_{f}=0$

with boundary conditions (13) and (14) becomes,

$u_{f}=0, \theta_{f}=1, \phi_{f}=1, \frac{\partial^{2} u_{f}}{\partial y^{2}}=0$ at $y=1$

$u_{f}=0, \theta_{f}=0, \phi_{f}=0, \frac{\partial^{2} u_{f}}{\partial y^{2}}=0$ at $y=0$

Solving equations (16) - (18) subject to conditions (19) and (20) and using equations (15), we get

$$
\begin{aligned}
& \theta(y, t)=\left(\frac{e^{m_{1} y}-e^{-m_{1} y}}{e^{m_{1}}-e^{-m_{1}}}\right) e^{i w t} \\
& \phi(y, t)=\left(\frac{e^{m_{2} y}-e^{-m_{2} y}}{e^{m_{2}}-e^{-m_{2}}}\right) e^{i w t} \\
& u(y, t)=\left[\begin{array}{l}
C_{1} e^{m_{3} y}+C_{2} e^{m_{4} y}+C_{3} e^{-m_{3} y}+C_{4} e^{-m_{4} y} \\
-Z_{2}-Z_{3}\left(e^{m_{1} y}-e^{-m_{1} y}\right)-Z_{4}\left(e^{m_{2} y}-e^{-m_{2} y}\right)
\end{array}\right] e^{i w t}
\end{aligned}
$$

where $m_{1}, m_{2}, m_{3}, m_{4}, C_{1}, C_{2}, C_{3}$ and $C_{4}$ are given in the Appendix.

The Shear stress $\left(C_{f}\right)$ at the upper wall of the channel is given by

$$
C_{f}=-\left.\frac{\partial u}{\partial y}\right|_{\text {at } y=1}=-\left[\begin{array}{l}
C_{1} m_{3} e^{m_{3}}+C_{2} m_{4} e^{m_{4}}-C_{3} m_{3} e^{-m_{3}}-C_{4} m_{4} e^{-m_{4}} \\
-Z_{3} m_{1}\left(e^{m_{1}}+e^{-m_{1}}\right)-Z_{4} m_{2}\left(e^{m_{2}}+e^{-m_{2}}\right)
\end{array}\right] e^{i w t}
$$




\section{Effect of Hall Current in Oscillatory Flow of a Couple Stress Fluid in an Inclined Channel}

The rate of heat transfer or Nusselt Number $(N u)$ and the rate of mass transfer or Sherwood Number $(S h)$ at the upper wall of the channel are given by

$$
\begin{gathered}
N u=-\left.\frac{\partial \theta}{\partial y}\right|_{\text {at } y=1}=-\left[\frac{m_{1}\left(e^{m_{1}}+e^{-m_{1}}\right)}{\left(e^{m_{1}}-e^{-m_{1}}\right)}\right] e^{i w t} \\
S h=-\left.\frac{\partial \phi}{\partial y}\right|_{\text {at } y=1}=-\left[\frac{m_{2}\left(e^{m_{2}}+e^{-m_{2}}\right)}{\left(e^{m_{2}}-e^{-m_{2}}\right)}\right] e^{i w t}
\end{gathered}
$$

From equation (8), it is possible to arrive a relation for 'critical magnetic field' $\left(\mathrm{B}_{\mathrm{c}}\right)$, at which the $+v e$ velocity will change to $-v e$. Hence ' $B_{c}$ ' can be derived from the dimensionless parameter $H$ as,

$$
H^{2}=\frac{a^{2} \sigma B_{c}{ }^{2}}{\rho v}
$$

\section{Results and discussions}

We have taken the real part of the results obtained in equations (21) - (26) and made use of the following parameter values $P e=0.71, G r=1, G c=1, K c=1, S c=1, R e=1$, $t=0, w=1, S=1, H=1, N=1, \alpha=\pi / 4, m=1, B=1$, and $\gamma=1$. The said values are kept common in the entire study except for varied values as displayed in Figures 2 to 20.

Figure 2 shows the effect of Reynolds number $(R e)$ on velocity profile. Increase of small value of $R e$ does not show any effect in the velocity. But for $R e>10$, shows a small difference of increase in velocity. Figure 3 represents the effect of Peclets number $(\mathrm{Pe})$ on velocity profile. Higher values of $P e$ shows the higher values of velocity.

Figure 4 displays the thermal effect or heat radiation parameter $(N)$ on velocity profile. The thermal effect on velocity shows a remarkable role, ie, the increase of $N$ shows a velocity increase throughout the diameter of the channel $(y)$ and it is maximum at the midpoint, ie., it increases in the lower channel half space and decreases in the upper channel half space.

Figure 5 displays the effect of Hartmann number $(H)$ on velocity profile. A small increase on velocity follows Hartmann number. Our results show that increasing magnetic field intensity increases the shear stress which is contrary to Makinde and Mhone [3] that they observed a decreasing trend, but the increase in our result is very little, in the order of $10^{-4}$.

Figure 6 shows the effect of Grashoff number $(G r)$ on velocity profile. A decrease in velocity maximum is observed when $y \approx 0.5$ and the variation of velocity maximum is same for the interval of $G r=0.5,1.0$ and 1.5 respectively, (at $\mathrm{y}=0.5$, $\mathrm{u}_{\max }=0.0053$ for $G r=1.0$; at $\mathrm{y}=0.4, \mathrm{u}_{\max }=0.00304$ for $\mathrm{Gr}=1.5$ ). That is, when $\mathrm{Gr}>1.0$, the $\mathrm{u}_{\max }$ is obtained at $\mathrm{y}<0.5$, ie., at the lower half of the channel, which can also be expessed as, with increase of Gr the resistivity also increases which is similar to the result obtained by Ahmed Sahin et al.[3]. 
Nirmala P. Ratchagar, Balakrishnan and Vasanthakumari

Figure 7 displays the effect of modified Grashoff number $(G c)$ on velocity profile. A decrease in velocity due to increase in $G c$ is predicted. Figure 8 shows the effect of couple stress fluid $(\gamma)$ on velocity profile. Our result also shows an increase in the couple stress parameter increases the resistance to the flow and thereby which may lead to decrease of the volume rate flow, which is in coincidence with Pal et al. [16]. The velocity peak is observed at the midpoint of $y$ and it is directly proportional to the couple stress parameter $(\gamma)$, but it is inversely proportional to the angle of inclination $(\alpha)$ and are shown in Figures 8 and 9.

The role of the Hall current in increasing the velocity of the fluid is critically essential, since the limit at which the influence of magnetic field in the absence of Hall current shows a negative velocity. It can also be stated that the inclusion of Hall current alone gives a meaningful argument about the physical meaning of velocity in this study. From the Figure.10 it is shown that increase of magnetic field increases the velocity maximum, but, when $H>9.3$, it gives a negative velocity (Figure.11), in the absence of Hall current $(m=0)$. And at the presence of Hall current $(m>0)$, the velocity is always positive for all external magnetic field. Hence, for the possible increase of velocity and to obtain the velocity maximum at the center of the channel, presence of Hall current is an essential one.

It is important to note here that, at the first time, the limit of magnetic field for the continuous application of it in the dynamics of the fluid is determined. We have arrived here a 'Critical Magnetic Field', ' $B_{c}$ ', that is, the magnetic field at which the velocity becomes zero (eqn. 27), which is pictorially shown in Figure.12. From the results it is evidenced that the forward movement of the fluid beyond ' $\mathrm{B}_{\mathrm{c}}$ ' is possible only because of the presence of Hall current.

Figure 13 shows the effect of Peclets number $(P e)$ on temperature profile. When $y$ increases the temperature effect increases linearly, but when the Peclets number increases the slope get decreases. A similar effect is also observed for increase of heat radiation parameter $(N)$, which is shown in Figure 14.

When the chemical reaction parameter $(K c)$ increases, the concentration decreases slightly, which is shown in Fig.15. And the concentration does not affect much due to the increase of $S c$ and hence higher values of $K c$ and $S c$ may contribute for higher concentration profile (Figure 16).

The variation of shear stress with porosity for different $K c$ values is plotted in Figure 17. A shift in shear stress for $K c$ values $(\approx 0.001)$ for the range of ' $S$ ' indicates the complete influence of $K c$ in it, but the gradual growth of shear stress for increasing $\mathrm{S}$ shows the difference of shear stress (slope $=0.00062 ; K c=1.0$ ) with $S$ is less influential than Kc. But the upward shift in shear stress with Hartmann number ' $H$ '(Figure 18) is comparatively high $(\approx 0.004)$ and the slope of 0.00030 for $\mathrm{N}=1.0$ shows the stiffness of shear stress values for $H$. From the Figures $17 \& 18$ it is conceived that the increasing radiation parameter through heat absorption causes an increase in the magnitude of shear stress, which agrees well with Ref.9.

The Nusselt number decreases when $P e$ and $N$ increases(Figure 19) and remains constant for other parametric changes. The change of Nusselt number ' $N$ ' with ' $P e$ ' is gradual for a particular thermal radiation parameter ' $N$ '. For eg., if $N=1.0$, the slope obtained is 0.09606 in the downstream, but the higher $N$ values shifts the Nusselt number downwards with increasing displacement and hence the greater influence of $\mathrm{N}$ in Nusselt 
Effect of Hall Current in Oscillatory Flow of a Couple Stress Fluid in an Inclined Channel

number is understood. The Sherwood number also decreases when $S c$ and $K c$ increases (Figure 20). The change of Sherwood number ' $S h$ ' with ' $S c$ ' shows a gradual decreasing effect for a particular chemical reaction parameter ' $K c$ '. For eg., if $K c=1.0$, the slope obtained is 0.05489 in the downstream, but the higher $K c$ values shifts the Sherwood number downwards with nearly constant displacement and hence the chemical reaction parameter's negative influence on Sherwood number is explored.

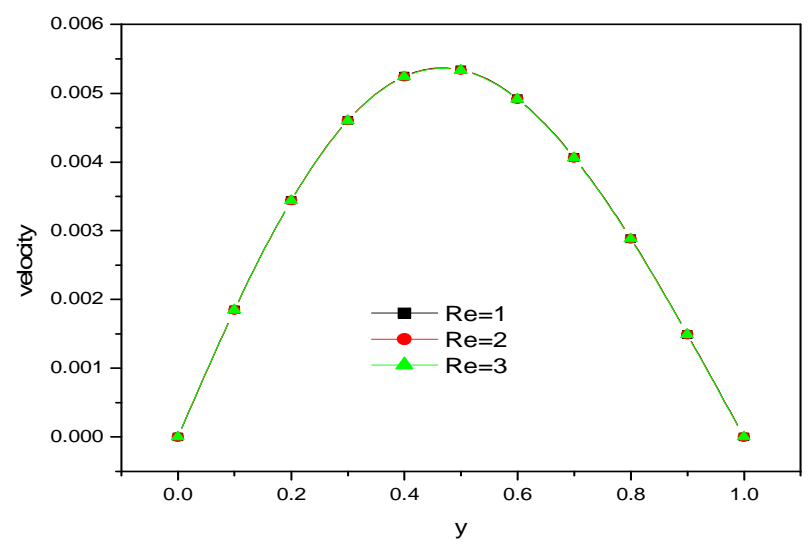

Figure 2: Variation of velocity for different values of ' $R e$ '.

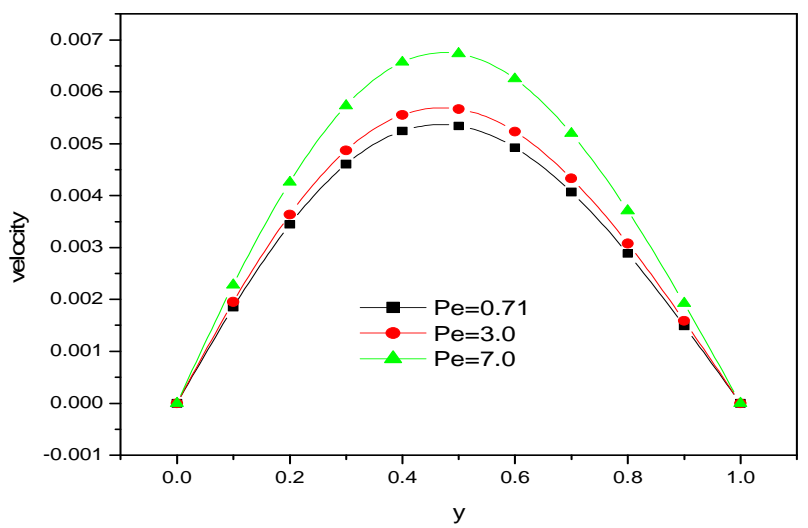

Figure 3: Variation of velocity for different values of ' $P e$ '. 
Nirmala P. Ratchagar, Balakrishnan and Vasanthakumari

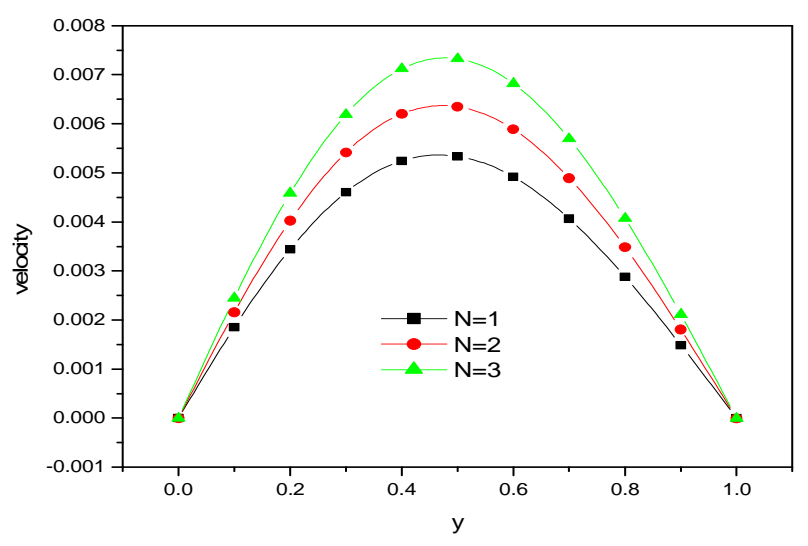

Figure 4: Variation of velocity for different values of ' $N$ '.

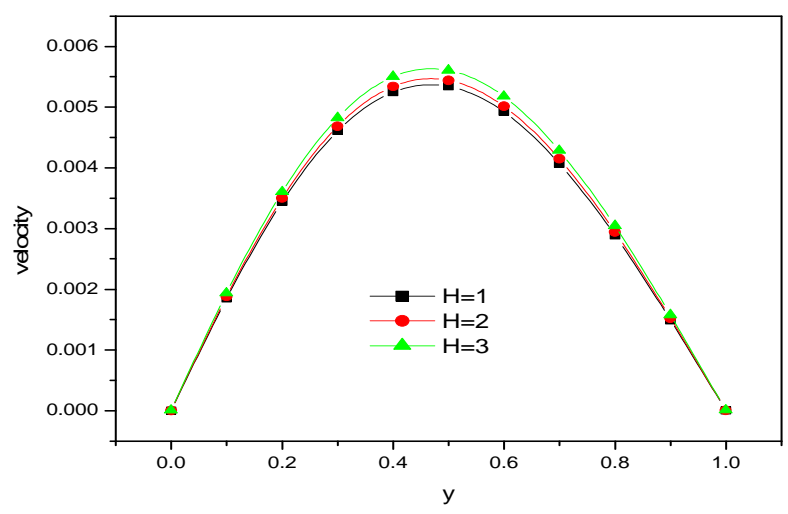

Figure 5: Variation of velocity for different values of ' $H$ '.

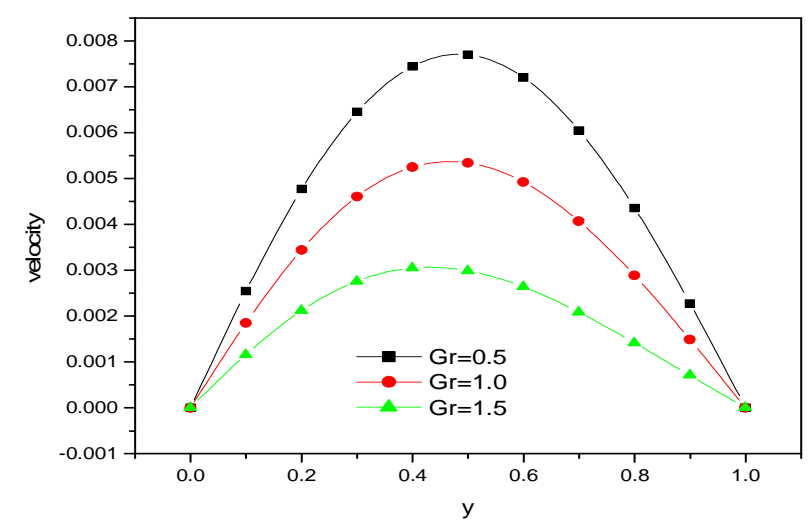

Figure 6: Variation of velocity for different values of ' $G r$ '. 
Effect of Hall Current in Oscillatory Flow of a Couple Stress Fluid in an Inclined Channel

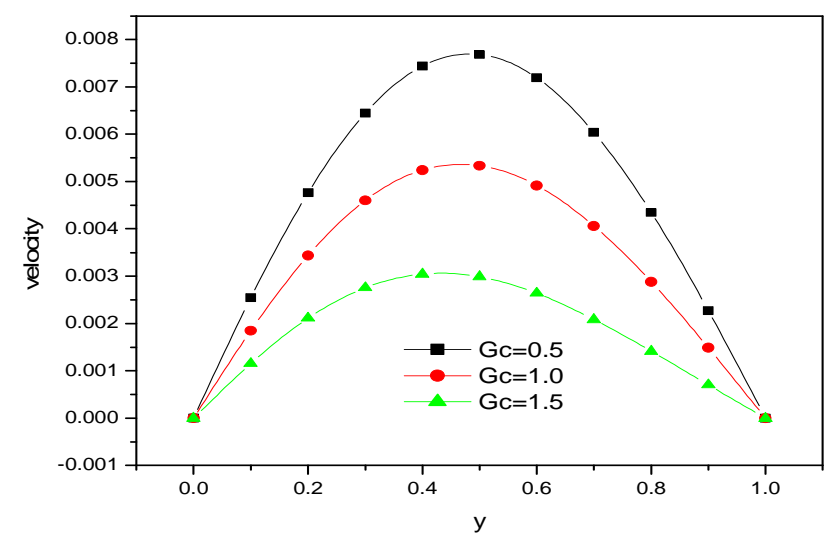

Figure 7: Variation of velocity for different values of ' $G c$ '.

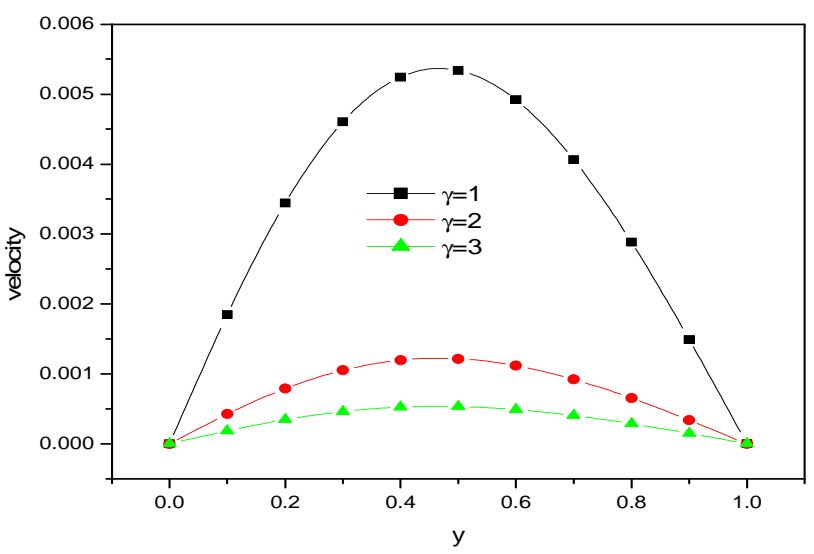

Figure 8: Variation of velocity for different values of ' $\gamma$ '.

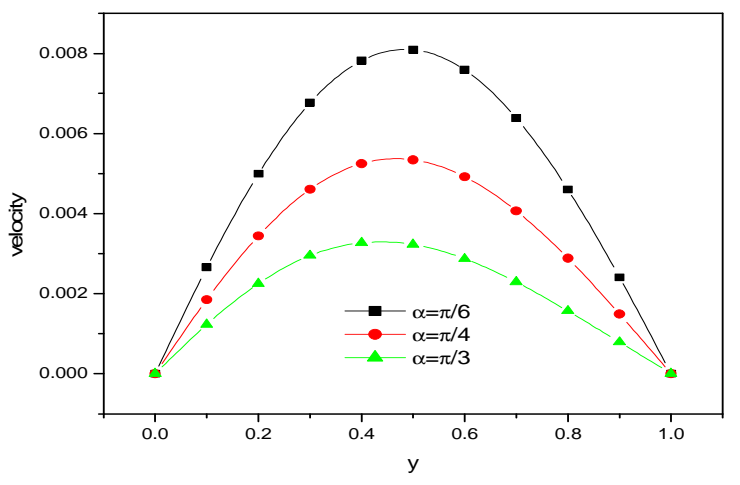

Figure 9: Variation of velocity for different values of ' $\alpha$ '. 
Nirmala P. Ratchagar, Balakrishnan and Vasanthakumari
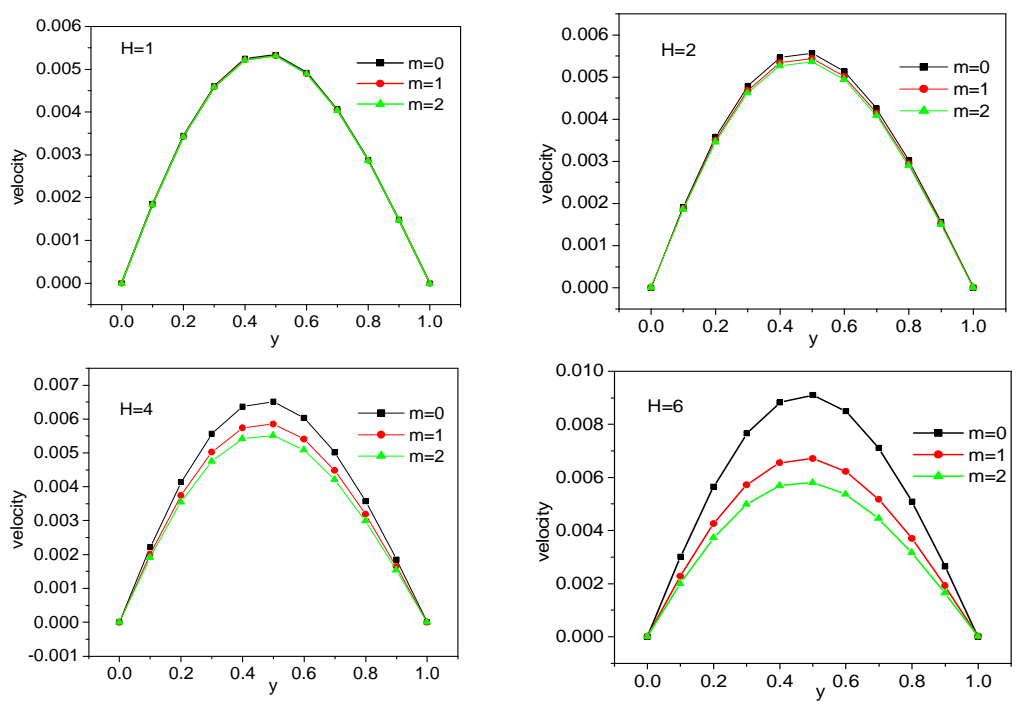

Figure 10: Influence of External Magnetic field in the velocity of the fluid.
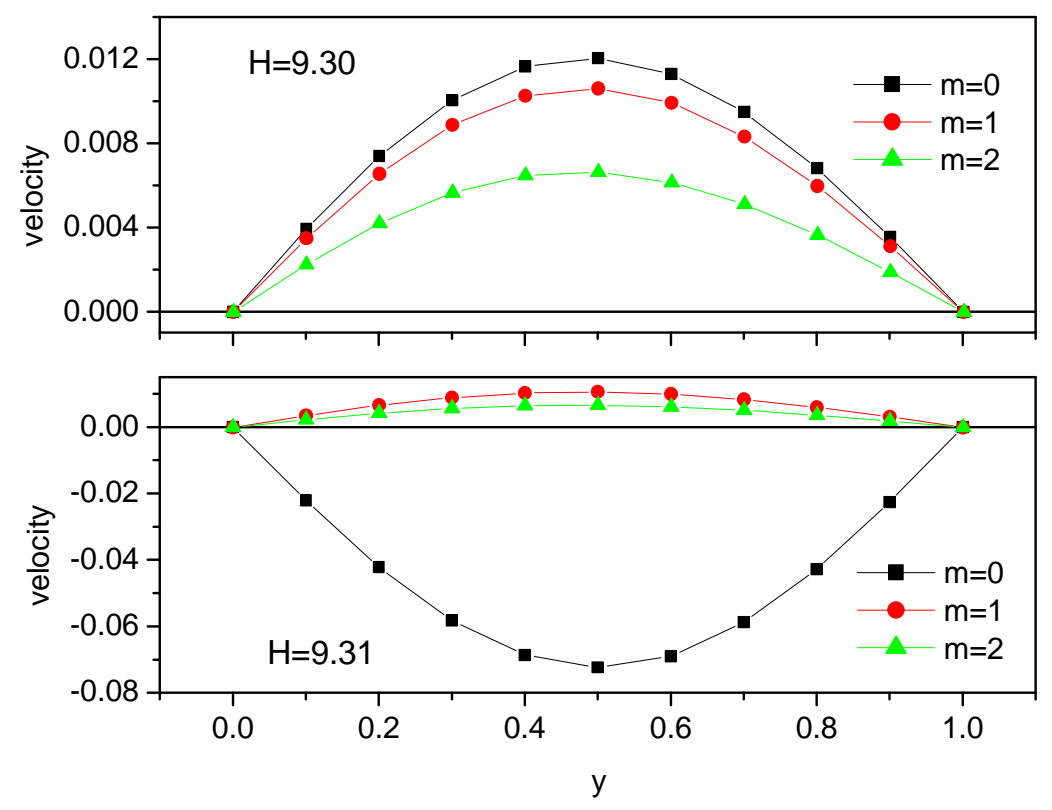

Figure 11: Limit of External Magnetic field for the positive fluid velocity. 
Effect of Hall Current in Oscillatory Flow of a Couple Stress Fluid in an Inclined Channel

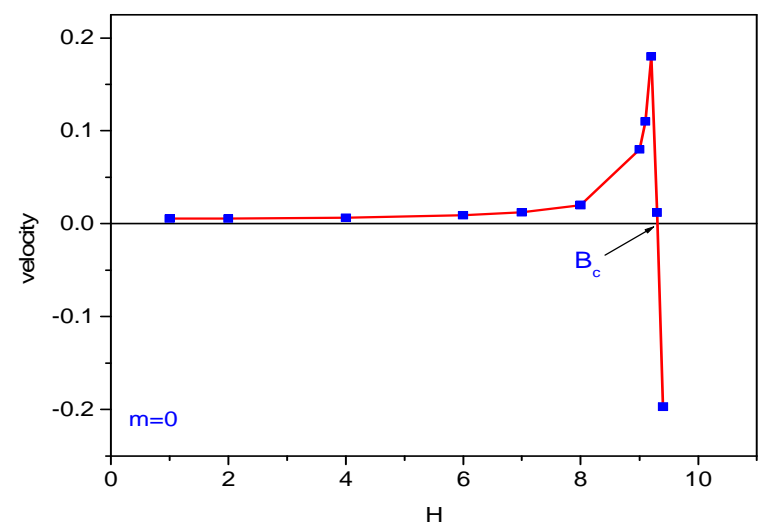

Figure 12: Determination of critical magnetic field, ' $\mathrm{B}_{\mathrm{c}}$ '.

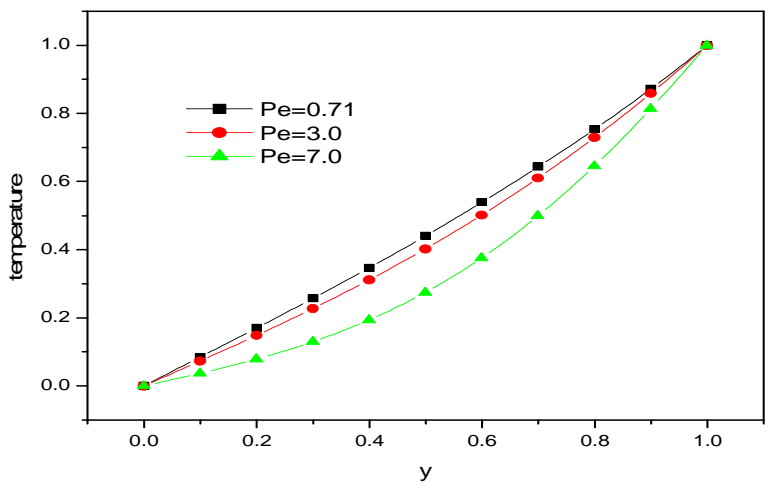

Figure 13: Variation of temperature for different values of ' $P e$ '.

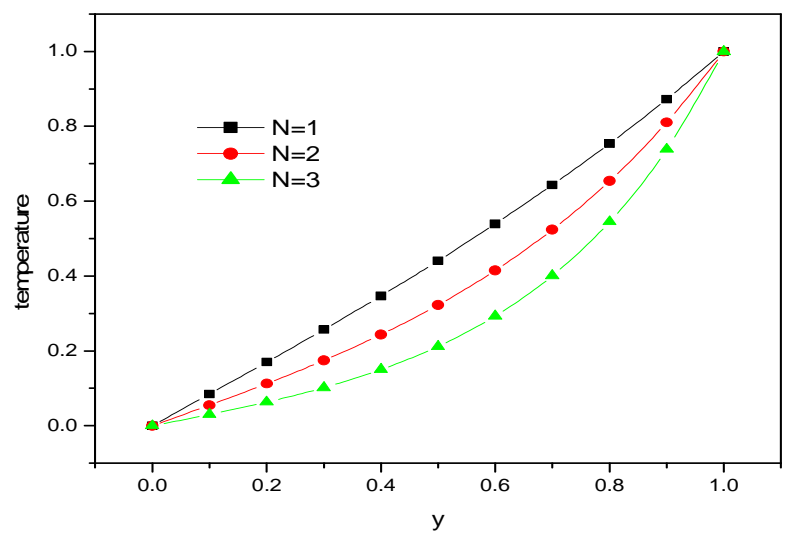

Figure 14: Variation of temperature for different values of ' $N$ '. 
Nirmala P. Ratchagar, Balakrishnan and Vasanthakumari

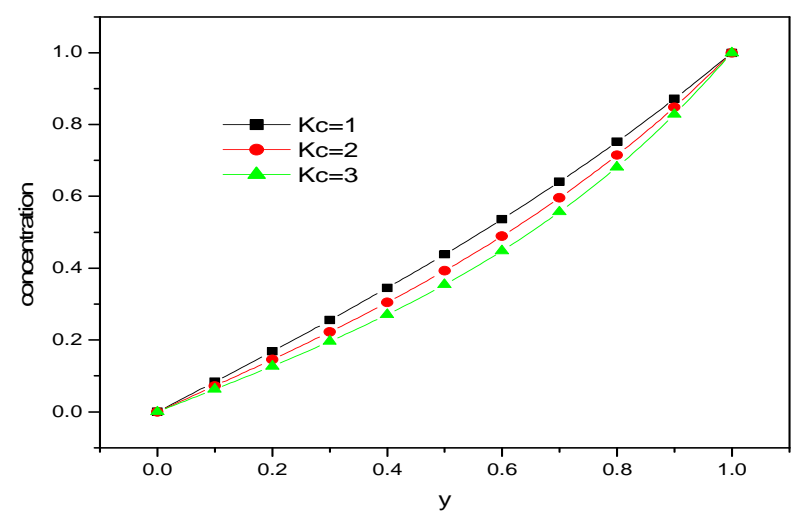

Figure 15: Variation of concentration for different values of ' $K c$ '.

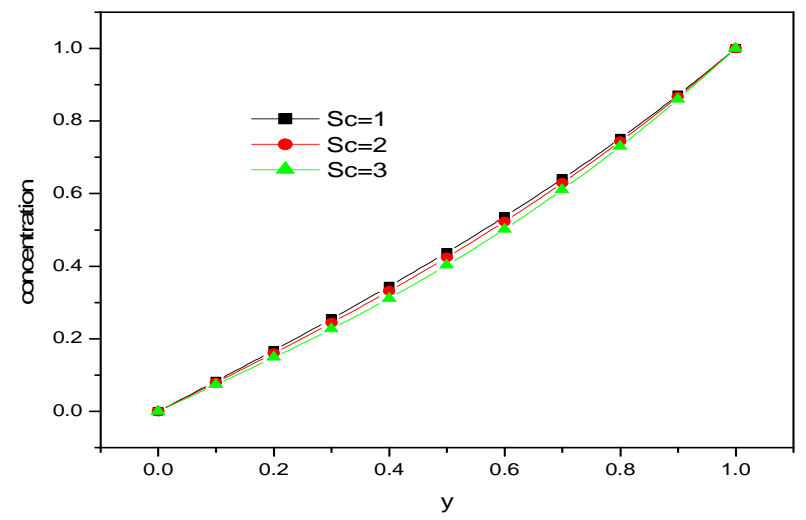

Figure 16: Variation of concentration for different values of ' $S c$ '.

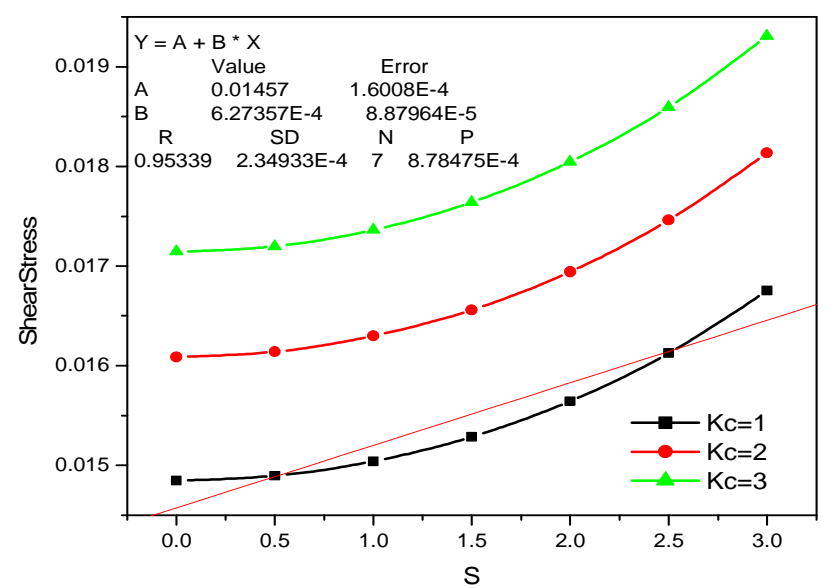

Figure 17: Effect of shear stress with porous parameter for different 'Kc'values 
Effect of Hall Current in Oscillatory Flow of a Couple Stress Fluid in an Inclined Channel

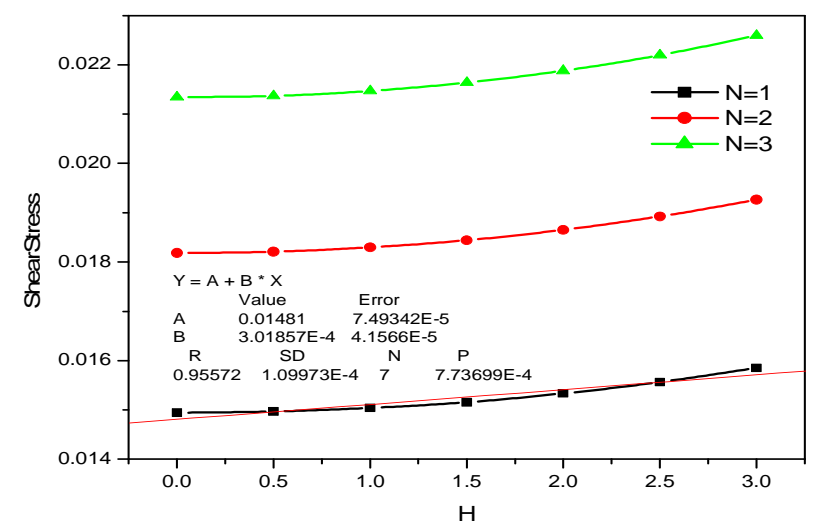

Figure 18: Variation of shear stress with Hartman No. for different values of ' $N$ '.

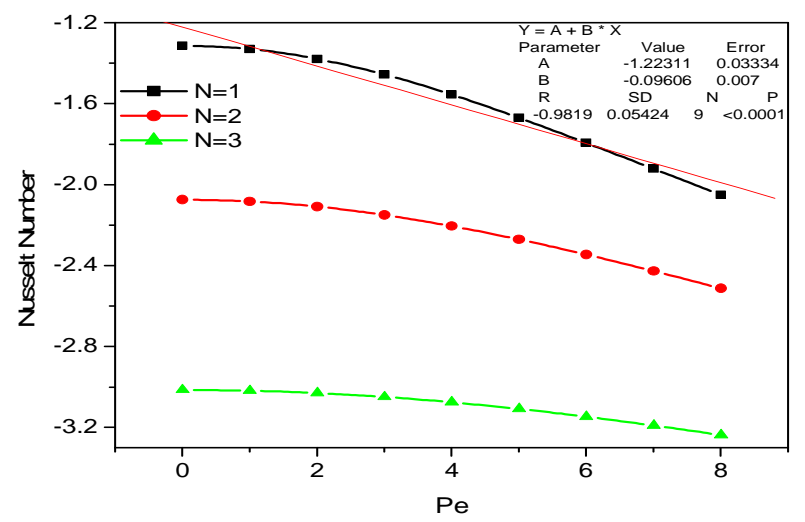

Figure 19: Variation of Nusselt Number with ' $P e$ ' for different values of ' $N$ '.

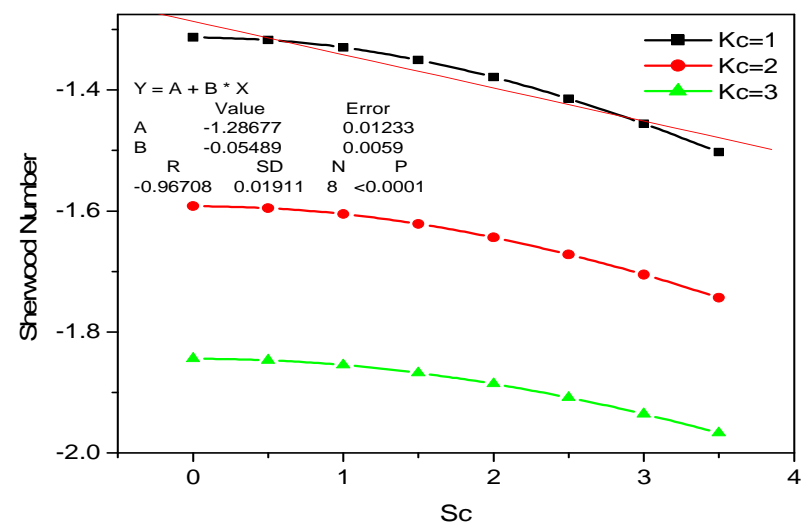

Figure 20: Variation of Sherwood Number with ' $S c$ ' for different values of ' $K c$ '. 
Nirmala P. Ratchagar, Balakrishnan and Vasanthakumari

\section{Conclusion}

This paper investigates the effect of hall current in oscillatory flow of a couple stress fluid in an inclined channel. The velocity and temperature profiles are obtained analytically and numerically using Mathematica and showed graphically for various parameters involved in the equations. The following findings are obtained out of this work.

- The velocity profile increased due to increase in $P e, N$, and $H$ while it decreases due to increase in $\alpha, G r, G c$, and $\gamma$.

- A decrease in temperature profile is function of an increase in $N$ and $P e$.

- A decrease in concentration profile is function of an increase in $K c$ and $S c$.

- The increasing radiation parameter through heat absorption causes an increase in the magnitude of shear stress.

- The rate of heat transfer decreases with increasing parameter $P e$ and $N$.

- The rate of mass transfer decreases with increasing parameter $S c$ and $K c$.

- Comparison of our results with the available literatures shows good coincidence, e.g., Ref. $(3,9,16)$.

- A negative velocity is expected beyond $H=9.3$ in the absence of Hall current.

- A critical Magnetic Field ' $B_{c}$ ' is introduced at the first time, and the limit of the Magnetic field for forward direction of the fluid is determined.

- The inclusion of Hall current can nullify the effect of ' $B_{c}$ ', ie., eliminating the reverse movement of blood flow.

Acknowledgement. The reviewer's comments and suggestions on this paper are highly useful to make this manuscript fruitful and the authors greatly acknowledge the reviewer for his/her support.

\section{REFERENCES}

1. N.Ali, S.U.Khan and Z.Abbas, Hydromagnetic flow and heat transfer of a Jeffery fluid over an oscillating stretching surface, Z. Naturforsch. A., 70 (2015) 567-576.

2. Ali Aamir and S.Asghar, Analytic solution for oscillatory flow in a channel for Jeffery fluid, J. Aerosp. Eng., 12 (2016) 1-12.

3. D.Ahmed Sahin, O.Anwar Beg, S.K.Ghosh, A couple stress fluid modeling on convection oscillatory hydromagnetic flow rotating channel, Ain Shams Engineering Journal, 5 (2014) 1249-1265.

4. A.C.L.Cogley, W.G.Vinvent, and E.S.Giles, Differential approximation for radiative heat transfer in non-linear equations-grey gas near equilibrium, American Institute of Aeronautics and Astronautics, 6 (1968) 551-553.

5. M.E.Erdogan, A note on unsteady flow of a viscous fluid due to an oscillating plane wall, Int. J. Non-linear Mech, 35 (2000) 1-6.

6. N.T.M.Eldabe, A.A.Hassan, M.A.A.Mohamed, Effects of couple stress on the MHD of Non-Newtonian unsteady flow between two parallel porous plates, Zeitshrift fur Naturforschung, 58 (2003) 204-210

7. P.S.Hiremath, and P.M.Patil, Free convection effects on the oscillatory flow of a couple stresses fluid through a porous medium, Acta Mech., (1993) 143-158. 
Effect of Hall Current in Oscillatory Flow of a Couple Stress Fluid in an Inclined Channel

8. Khem Chand, K.D.Singh and Shavnam Sharma, Effect of hall current and rotation on heat transfer in MHD flow of oscillating dusty fluid in a porous channel, Ind. J. Pure \& App. Phys., 51 (2013) 669-682.

9. O.D.Makinde and P.Y.Mhone, Studied Heat transfer to MHD oscillatory flow in a channel filled with porous medium, Rom. J. Phys., 50 (2005) 931-938.

10. Md.Mizanur Rahman, Md.Abdul Hye , Md.Mostafizur Rahman and Md.Mahafujur Rahaman, Numerical simulation on MHD free convection mass and heat transfer fluid flow over a vertical porous plate in a rotating system with induced magnetic field, Annals of Pure and Applied Mathematics, 7 (2014) 35-44

11. Md.Saidul Islam, Md.Samsuzzoha, Shamim Ara and Pinakee Dey, MHD free convection and mass transfer flow with heat generation through an inclined plate, Annals of Pure and Applied Mathematics, 3 (2013) 129-141

12. Mohamed Abdel-Wahed and Mohamed Akl, Effect of hall current on MHD flow of a nanofluid with variable properties due to a rotating disk with viscous dissipation and nonlinear thermal radiation, AIP ADVANCES, 6 (2016) 1-14

13. Nisat Nowroz Anika, Md.Mainul Hoqueand Nazmul Islam, hall current effects on magnetohydrodynamics fluid over an infinite rotating vertical porous plate embedded in unsteady laminar flow, Annals of Pure and Applied Mathematics, 3(2013)189-200.

14. A.Ogulu, On the oscillating plate-temperature flow of a polar fluid past a vertical porous plate in the presence of couple stresses and radiation, Int. Comm. HeatMass Transfer, 32 ( 2003) 1231-1243.

15. E Omokhuale and G I Onwuka, Effect of mass transfer and hall current on unsteady MHD flow of a viscoelastic fluid in a porous medium, IOSR Journal of Engineering, 2 (2012) 50-59.

16. D.Pal, N.Rudraiah, R.Devanathan, A couple stress model of blood flow in the microcirculation, Bull. Math. Biol., 50 (1988) 329-344.

17. D.Sarma, and K.K.Pandit, Effects of Hall current, rotation and Soret effects on MHD free convection heat and mass transfer flow past an accelerated vertical plate through a porous medium, to appear in Ain Shams Eng .J. http://dx.doi.org/10.1016/j.asej.2016.03.005.

18. M.S.Sarojini, M.V.Krishna, and C.U.Shankar, MHD flow of a couple stress fluid through a porous medium in a parallel plate channel in presence of effect of inclined magnetic field, Int. J. Phys.Math. Sci., 1 (2011) 9-18.

19. G. S. Seth, N.Nandkeolyar, Md S.Ansari, Effects of hall current and rotation on unsteady MHD coquette flow in the presence of an inclined magnetic field, Journal of Applied Fluid Mechanics, 5 (2012) 67-74.

20. K.D.Singh and Reena Pathak, Effect of rotation and hall current on mixed convection MHD flow through a porous medium filled in a vertical channel in presence of thermal radiation, Ind. J. Pure \& App. Phys., 50 (2012) 77-85.

21. V.M.Soundalgekar and A.G.Uplekar, IEEE Transactions on Plasma Science, 14 (1986) 579-583.

22. V.K.Stokes, Couple stresses in fluids, 9 (1966) 1709-1715.

23. M.Syamala Sarojini, M. Veerakrishna, C.Umashankar, MHD flow of a couple stress fluid through a porous medium in a parallel plate channel in presence of effect of inclined magnetic field, Int. J. of Physics and Mathematical Sciences, 1(2011), 9-18. 
Nirmala P. Ratchagar, Balakrishnan and Vasanthakumari

24. K.Vajravelu and J.Rivera, Hydromagnetic flow at an oscillating plate, Int. J. Nonlinear Mech, 38 (2003) 305-312.

25. M.Veerakrishna and Jagdish Prakash, Hall current effects on unsteady MHD flow in a rotating parallel plate channel bounded by porous bed on the lower half-darcy lapwood model, Open Journal of Fluid Dynamics, 5 (2015) 275-294.

26. M.Veerakrishna and B.V.Swarnalathamma, Hall effects on unsteady MHD free convection flow of an incompressible electrically conducting second grade fluid through a porous medium over an infinite rotating vertical plate fluctuating with heat source/sink and chemical reaction, IOSR Journal of Mathematics, 12 (2016) 8-24. 
Effect of Hall Current in Oscillatory Flow of a Couple Stress Fluid in an Inclined Channel

$$
\begin{aligned}
& \text { Appendix: } \\
& m_{1}=\sqrt{N^{2}-i w P e} \\
& m_{2}=\sqrt{S c i w+K c} \\
& m_{3}=\sqrt{\frac{\frac{-1}{\gamma^{2}}+\sqrt{\frac{-1}{\gamma^{4}}+\frac{4 Z_{1}}{\gamma^{2}}}}{2}} \\
& m_{4}=\sqrt{\frac{\frac{-1}{\gamma^{2}}-\sqrt{\frac{-1}{\gamma^{4}}+\frac{4 Z_{1}}{\gamma^{2}}}}{2}} \\
& Z_{1}=\operatorname{Re} i w+S^{2}+\frac{H^{2}}{1+m^{2}} \\
& A_{i}=\gamma^{2} m_{i}^{4}+m_{i}^{2}-Z_{1} ; i=1,2 \\
& Z_{2}=\frac{B}{Z_{1}} \\
& Z_{3}=\frac{G r \sin \alpha}{A_{1}\left(e^{m_{1}}-e^{-m_{1}}\right)} \\
& Z_{4}=\frac{G c \sin \alpha}{A_{2}\left(e^{m_{2}}-e^{-m_{2}}\right)} \\
& Z_{5}=Z_{3}\left(e^{m_{1}}-e^{-m_{1}}\right) \\
& Z_{6}=Z_{4}\left(e^{m_{2}}-e^{-m_{2}}\right) \\
& Z_{7}=Z_{3} m_{1}^{2}\left(e^{m_{1}}-e^{-m_{1}}\right) \\
& Z_{8}=Z_{4} m_{2}^{2}\left(e^{m_{2}}-e^{-m_{2}}\right) \\
& C_{1}=-C_{2}-C_{3}-C_{4}+Z_{2} \\
& C_{2}=-C_{4}+\frac{Z_{2} m_{3}^{2}}{\left(m_{3}^{2}-m_{4}^{2}\right)} \\
& C_{3}=\frac{-1}{\left(e^{m_{3}}-e^{-m_{3}}\right)}\left[C_{2}\left(e^{m_{3}}-e^{m_{4}}\right)+C_{4}\left(e^{m_{3}}-e^{-m_{4}}\right)-Z_{2}\left(e^{m_{3}}-1\right)+\left(Z_{5}+Z_{6}\right)\right] \\
& C_{4}=\frac{Z_{2} m_{3}^{2}\left(1-e^{m_{4}}\right)+\left(Z_{5}+Z_{6}\right) m_{3}^{2}-\left(Z_{7}+Z_{8}\right)}{\left(m_{3}^{2}-m_{4}^{2}\right)\left(e^{-m_{4}}-e^{m_{4}}\right)}
\end{aligned}
$$

